\title{
A New Algorithm about Market Demand Prediction of Automobile
}

\author{
Zhiming Zhu ${ }^{1}$, Tao Chen $^{2} \&$ Tianmiao Shen ${ }^{2}$ \\ ${ }^{1}$ Business School, Hohai University, China \\ ${ }^{2}$ School of Management \& Engineering, Nanjing University, China \\ Correspondence: Zhiming Zhu, West Focheng Road No. 8, Jiangning District, Hohai University, Boxue building, \\ Nanjing, Jiangsu, China. E-mail: zhimingzhu@126.com
}

Received: April 23, 2014 Accepted: May 12, $2014 \quad$ Online Published: July 28, 2014

doi:10.5539/ijms.v6n4p100 URL: http://dx.doi.org/10.5539/ijms.v6n4p100

\begin{abstract}
An extensive evaluation hierarchy model of automobile short-term demand was established to prevent the disadvantages of previous models mainly for single time series. The definition of extensive correlation evaluation was proposed, and then the method was discussed to reflect the correlation of factors on automobile demand. Utilizing extensive skills, factors and sub-factors were represented as correlation eigenmatrix which could ensure the level of each factor's influences on automobile demand. Then short-term historical data was predicted while it was compared with existing data, the results show that the predictive error is less than $6 \%$, which confirms the validation of predictive model. This study provides the foundations for government's macroeconomic control and automobile manufacturers' production.
\end{abstract}

Keywords: automobile, short-term demand, prediction, extensive correlation evaluation, continuous time model

\section{Introduction}

The demand analysis of automobile is mainly characterized by the following features: there are too many affecting factors on demand changes, the factors are very complex, and the relationship between the primary and secondary variables is changeful which is difficulty in quantitative analysis accurately; Meanwhile the data quantity involved in demand analysis is large, at the same time, the requirements for the timeliness of the analytical algorithm and arithmetic operation cost is high ( $\mathrm{Su}, 2010)$. During the process of research, we find that when forecasting the demand, our domestic automobile manufacturers usually use simple analysis tool with backward methods; what is more, they also analyze the quantitative and qualitative separately. Usually the uncertainty of demand often makes prediction conclusion which drew from the modeling method unsatisfactory. Therefore, it is imperative for us to establish an effective auto demand forecasting model. The key point to the implementation of scientific prediction depends on whether the qualitative method and the quantitative method are connected closely, which requires to treat the quantitative method as a foundation and comprehensively analyze the predicted object qualitatively (Bao, 2011). Hence, the paper proposes a dynamic continuous time series prediction model based on qualitative analysis. This model consists of two parts, one is quantitative forecast model based on dynamic continuous time model, in which we fit new sequence by taking advantage of the dynamic continuous time model and learn about the historical data of demand, and give the quantitative prediction of product demand. The other is the extensive hierarchy analysis and evaluation model of influential factors based on product demand, concluding the correlation index model about the influential factors through the analysis of the influential factors about automobile market demand. This paper combines the two models from each part, establishes a final prediction model through quantitative model which based on qualitative analysis, and gives a more objective prediction result according to that.

\section{Time Continuous Model of Dynamic System}

\subsection{Time Continuous Model of Dynamic System}

Owing to our country's macro-control on automobile industry, it's hard to establish a prediction model of short-term car sales trends by traditional prediction method. The established prediction models such as ARIMA, ANN, Holt-winter, all of these, on the basis of a large amount of historical data, searching for the causalities which influence the future trend, so that they could forecast by establishing related extrapolation type model (Zuo \& Gao, 2011). The essence of car sales forecast is to study the time variation law of a dynamic system, which is to deduce the future changes of the system according to the existing status data. And the dynamic 
system model can be expressed by a high order differential equation. If the dynamic behaviors of car sales are observed by continuous time, we call it time continuous model of dynamic system. According to the features of short-term changes of auto sales, the rule of accumulative changes of sales can be expressed by $n$-th order differential equation (Zhao, 1991).

According to time continuous model of dynamic system put forward by Shipeng Zhao, we can express it with $\mathrm{n}$-th order differential equation:

$$
\frac{d^{n} x_{1}}{d t^{n}}+\sum_{i=1}^{n} a_{i} \frac{d^{n-1} x_{1}}{d t^{n-1}}=b_{0}+\sum_{i=1}^{h-1} b_{i} x_{i+1}
$$

Abbreviated as $D(n, h)$, where $x_{i}(i=1,2, \cdots, h-1)$ is accumulated time series of primitive time series

The following three mathematical models are going to be used:

First, 1st order linear dynamic model with single sequence DM $(1,1)$ model:

$$
\frac{d x_{1}^{(1)}}{d t}+\mathrm{ax}{ }_{1}^{(1)}=\mu
$$

where $\mathrm{a}, \mu$ are constant.

Second, 1 st order linear dynamic model with two sequences (DM $(1,2)$ model:

$$
\frac{d x_{1}^{(1)}}{d t}+\mathrm{a} x_{1}^{(1)}=b x_{2}^{(1)}
$$

Where $x_{2}{ }^{(1)}$ is once accumulated sequence of $\left\{x_{2}{ }^{(0)}\right\}$ series, a and $\mathrm{b}$ are constant.

Third, 1st order linear dynamic model with three sequences (DM $(1,3)$ model:

$$
\frac{d x_{1}^{(1)}}{d t}+a x_{1}^{(1)}=b x_{2}^{(1)}+c x_{3}^{(1)}
$$

Where $x_{3}{ }^{(1)}$ is once accumulated sequence generated from sequence $\left\{x_{3}{ }^{(0)}\right\}, \mathrm{a}, \mathrm{b}$ and $\mathrm{c}$ are constant.

\subsection{Data Processing}

Once accumulated generating operation process data before we establish fluctuant economic sequence model, while an inverse accumulated generating operation is usually used to restore the predicted value generated from the previous model. Economic sequence $X^{(0)}$ is nonnegative sequence (i.e., each component $x^{(0)}(k) \geq 0$ ), thus $X^{(1)}$ is monotone increasing. Liu Zhu points out that after accumulated generating operation sequence $X^{(1)}$ possesses better properties than the original sequence $X^{(0)}$, i.e., it has better smoothness, and fluctuant sequence generates monotone new sequence, which weakens the randomness of sample value sequence (Liu, 2009). Through accumulated generating operation, time series $\left\{x^{(0)}(t)\right\}, x^{(0)}(t) \geq 0, t=1,2, \cdots, n$ get monotone increasing $x^{(1)}(k)=\sum_{t=1}^{k} x^{(0)}(t)$. The original time series is nonnegative, and its data amplitude changes irregularly, but the generated time series is not only nonnegative, but also monotone increasing, namely data amplitude change has certain regularity. Compared with the original sequence, generated sequence has an enhanced determinacy. Derivable everywhere is the characteristic of smooth continuous function, but 
sequence $\left\{x^{(0)}(t)\right\}$ consists of discrete single points, thus usually it has no derivative, therefore we can't research smoothness of sequence $\left\{x^{(0)}(t)\right\}$ with derivative. We study the characteristics of smooth continuous function by the following points of view.

Hypothesis 1: if a sequence has roughly similar characteristics with smooth and continuous function, the sequence is considered to be smooth.

Definition 1: assume that $X(t)$ is continuous function defined on $[a, b]$, insert interior points on $[a, b]$.

$$
a=t_{1}<t_{2}<\cdots<t_{n}<t_{n+1}=b
$$

Now on $[a, b]$ there is a division $\Delta t_{k}=\left[t_{k}, t_{k+1}\right], k=1,2, \cdots, n$. We use $\Delta t_{k}$ to express the length of $\left[t_{k}, t_{k+1}\right], \Delta t_{k}=t_{k+1}-t_{k}, k=1,2, \cdots, n$.Take any point on $\left[t_{k}, t_{k+1}\right]$, we get $x(k)$, thus we have series $X=(x(1), x(2), \cdots, x(n)), X_{0}=\left(x\left(t_{1}\right), x\left(t_{2}\right), \cdots, x\left(t_{n}\right)\right)$ is recorded as lower boundary point sequence. Let $\Delta t=\max _{1 \leq k \leq n}\left\{\Delta t_{k}\right\}$, assume that $\mathrm{d}$ is a distance function in $\mathrm{n}$-dimensional space, $X *$ is the representative sequence for specified function. No matter how time zone $[a, b]$ is divided and how interior point in small time interval is selected, when $\Delta t \rightarrow 0$ there are:

(1) for any interior point sequence $X_{i}, X_{j}, d\left(X^{*}, X_{i}\right)=d\left(X^{*}, X_{j}\right)$;

(2) $d\left(X^{*}, X\right)=d\left(X^{*}, X_{0}\right)$

Than we call $x(t)$ smooth continuous function.

Theorem 1: assume a sequence $X=(x(1), x(2), \cdots, x(n), x(n+1)), Z$ is a mean sequence generated from $X, Z=(z(1), z(2), \cdots, z(n))$, among which $z(k)=0.5 x(k)+0.5 x(k+1), k=1,2, \cdots, n \cdot X^{*}$ is a representative sequence for a derivative function, and $\mathrm{d}$ is a distance function in $\mathrm{n}$-dimensional space. We still call it $\mathrm{X}$ after deleting $x(n+1)$ from $\mathrm{X}$, if the following conditions is satisfied, we call $X$ smooth sequence.

(1) $\mathrm{K}$ is sufficiently large, $x(k)<\sum_{i=1}^{k-1} x(i)$;

(2) $\max _{1 \leq k \leq n}\left|x^{*}(k)-x(k)\right| \geq \max _{1 \leq k \leq n}|x *(k)-z(k)|$.

(1), (2) is called sequence smoothness conditions

Theorem 2: assume that $X^{(0)}$ is nonnegative sequence, $X^{(0)}=\left(x^{(0)}(1), x^{(0)}(2), \cdots, x^{(0)}(1)\right)$, $x^{(0)}(k) \geq 0$ and $x^{(0)}(k) \in[a, b] k=1,2, \cdots, n \cdot X^{(r)}=\left(x^{(r)}(1), x^{(r)}(2), \cdots, x^{(r)}(n)\right)$ is $\mathrm{r}$-th accumulated 
generating time sequence of $X^{(0)}$, when $r$ is sufficiently large, for any $\varepsilon>0$, there exist $N, N<k \leq n$, the following equation is established $\frac{x^{(r)}(k)}{\sum_{i=1}^{k-1} x^{(r)}(i)}<\varepsilon$,that is, for the bounded nonnegative sequence, after many times accumulated generating operation ,the generated sequence is sufficiently smooth ,and smooth ratio $\rho(k) \rightarrow 0(k \rightarrow \infty)$

Theorem 3: assume that $X^{(0)}$ is a nonnegative sequence, $X^{(0)}=\left(x^{(0)} x(1), x^{(0)}(2), \cdots, x^{(0)} x(n)\right)$, $x^{(0)}(k) \geq 0 \quad$ and $\quad x^{(0)}(k) \in[a, b], \quad k=1,2, \cdots, n . X^{(1)}=\left(x^{(1)}(1), x^{(1)}(2), \cdots, x^{(1)}(n)\right) \quad$ is $\quad$ once accumulated generating sequence of $X^{(0)}, Z^{(1)}=\left(z^{(1)}(1), z^{(1)}(2), \cdots, z^{(1)}(n)\right)$, where $z^{(1)}(k)=0.5 x^{(1)}(k)+0.5 x^{(1)}(k-1)$ is adjacent mean generated sequence of $X^{(1)}$, then for any $\varepsilon_{1} \leq \varepsilon_{2} \in[0,1]$, there is a positive integer $N$,and for any $k$, $N<k \leq n$, there are

$$
\rho(k)=\frac{x^{(0)}(k)}{\sum_{i=1}^{k-1} x^{(0)}(i)}<\varepsilon_{1} \quad \frac{x^{(0)}(k)}{z^{(1)}(k)}<\varepsilon_{2}
$$

Both the accumulated generating operation and the mean generation can improve the smoothness of the sequence, we can use two kinds of methods through combination, even if we implement a mean generation after accumulated generating operation.

\section{Hierarchy Evaluation Model of Influential Factors}

\subsection{Factors Affecting the Auto Market}

The following factors affect the future trends of the automotive market and industry: political factors, economic factors, social factors and technical factors. Through the analyses to China's auto industry in the past, present and future, we find that: political factor is the guarantee for healthy development of automotive industry; economic factor is the foundation for the automotive industry to take off; technical factor is the prerequisite for prosperity of the automotive industry; social factors is the key to create the automotive consumer market (Feng, 2004; Wang \& Ma, 2006). According to the relevance of factors that affect the demand for cars (Cai, 1999), we establish a hierarchy evaluation model of automobile demand factors as follows: 


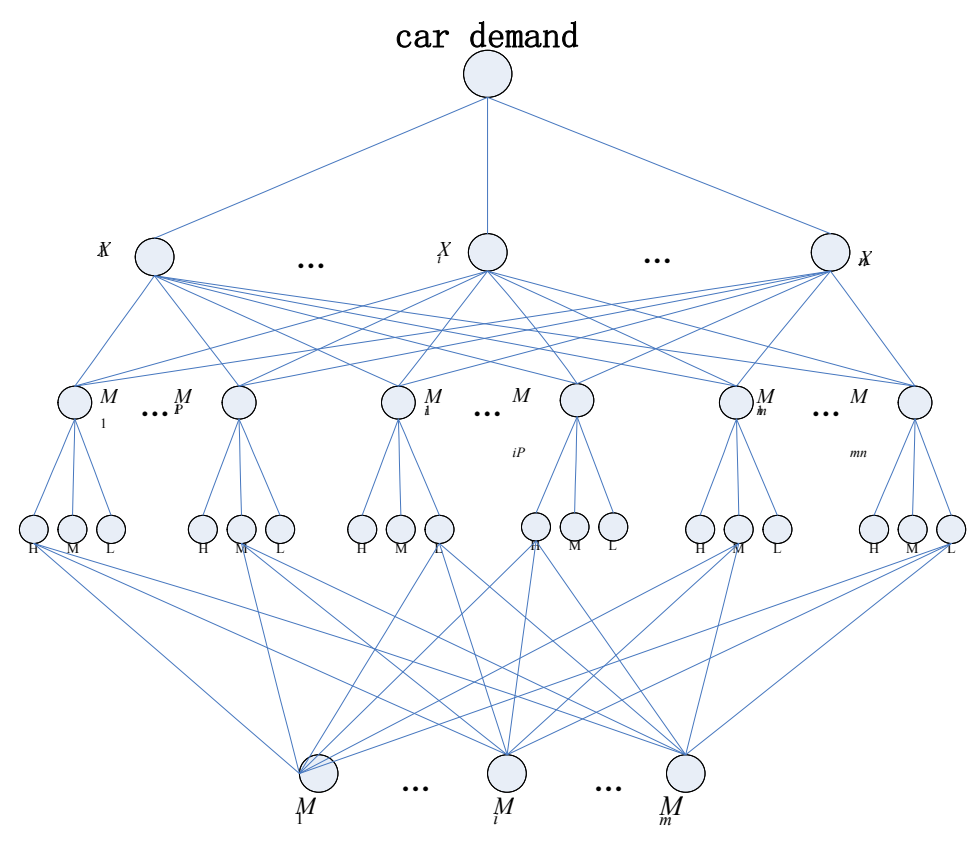

Figure 1. Car demand influential factors evaluation model

Where $M_{i},(i=1,2, \cdots, m)$ represent macro influential factors, for instance economic, social, technological and other factors affecting the demand for cars, $M_{i 1}, M_{i 2}, \cdots, M_{i P}$ represent the sub-factors that affect macro factors. $H, M, L$ respectively represent high, medium and low degree of the influence of sub-factors. $X_{1}, X_{2}, \cdots X_{n}$ indicate different types of automobiles.

Rule 1: rules to establish hierarchy evaluation model

(1) In the functional hierarchy, a complex system is decomposed in accordance with the basic relationship among its component elements;

(2) The highest level in hierarchy contains only one element: the overall goal. Other levels can contain a number of elements;

(3) There is no limit for levels in the hierarchy;

(4) When favoring comparison can not be carried out between certain elements, an appropriate new level must be inserted.

(5) Hierarchy is flexible, and can be modified at any time in order to adapt to the new guidelines.

\subsection{Evaluation Model Based on Analytic Hierarchy Process}

If $R$ represents the eigenmatrix of car demand influential factors, $M$ represents the factors affecting the demand for automobiles, $c$ represents various moments, $\mu(v)$ is membership degree of $v$, here $v$ is the corresponding value of $c$ (the influential factors of the demand for automobiles). Assume that $\mathrm{M}$ possesses $\mathrm{m}$ factors, and matrix consists of their shared n moments $\mu\left(v_{x j}\right)(x=1,2, \cdots, m ; j=1,2, \cdots, n)$, the following matrix called the eigenmatrix of automobile demand.

$$
R_{m n}=\left[\begin{array}{ccccc} 
& M_{1} & M_{2} & \cdots & M_{m} \\
c_{1} & \mu\left(v_{11}\right) & \mu\left(v_{21}\right) & \cdots & \mu\left(v_{m 1}\right) \\
c_{2} & \mu\left(v_{12}\right) & \mu\left(v_{22}\right) & \cdots & \mu\left(v_{m 2}\right) \\
\vdots & \vdots & \vdots & \cdots & \vdots \\
c_{n} & \mu\left({ }_{1 n}\right) & \mu\left(v_{2 n}\right) & \cdots & \mu\left(v_{m n}\right)
\end{array}\right]
$$

Definition 2: Establish sequential eigenmatrix $R_{f}$ for cars demand forecast and real sequential eigenmatrix $R_{r}$ which includes multiple influential factors. Where $x_{f i}(j), i=1,2, \cdots, n ; j=1,2, \cdots, m$ represents the time 
series for cars demand prediction, and $x_{r i}(j), i=1,2, \cdots, n ; j=1,2, \cdots, m$ represents the actual time series, we have

\subsection{Correlation Right Eigenmatrix}

$$
\begin{gathered}
R_{r}=\left[\begin{array}{ccccc} 
& M_{1} & M_{2} & \cdots & M_{n} \\
c_{1} & x_{r 1}(1) & x_{r 2}(1) & \cdots & x_{r n}(1) \\
c_{2} & x_{r 1}(2) & x_{r 2}(2) & \cdots & x_{r n}(2) \\
\vdots & \vdots & \vdots & \vdots & \vdots \\
c_{m} & x_{r 1}(m) & x_{r 2}(m) & \cdots & x_{r n}(m)
\end{array}\right] \\
R_{f}=\left[\begin{array}{ccccc} 
& M_{1} & M_{2} & \cdots & M_{n} \\
c_{1} & x_{f 1}(1) & x_{f 2}(1) & \cdots & x_{f n}(1) \\
c_{2} & x_{f 1}(2) & x_{f 2}(2) & \cdots & x_{f n}(2) \\
\vdots & \vdots & \vdots & \vdots & \vdots \\
c_{m} & x_{f 1}(m) & x_{f 2}(m) & \cdots & x_{f n}(m)
\end{array}\right]
\end{gathered}
$$

\subsubsection{Characteristics of One-Layer Correlation Right}

If $R_{w}$ represents weight matrix influencing all factors of each predictive object and $w_{i}(i=1,2, \cdots, n)$ represent the weights of $i$-th factor of each predictive object. Then we have

$$
R_{w}=\left[\begin{array}{ccccc} 
& M_{1} & M_{2} & \cdots & M_{n} \\
w_{i} & w_{1} & w_{2} & \cdots & w_{n}
\end{array}\right]
$$

\subsubsection{Characteristics of Two-Layer Correlation Right}

Demand for cars contains multi-layer influential factors, hence its corresponding weight should be divided into several layers. Take two layers as a sample, the rest can be done in the same manner, there is:

$$
R_{w}=\left[\begin{array}{ccccc} 
& M_{1} & M_{2} & \cdots & M_{n} \\
w_{i} & w_{1} & w_{2} & \cdots & w_{n} \\
& c_{11} c_{12} \cdots c_{1 k} & c_{21} c_{22} \cdot c_{2 k} & \cdots & c_{n 1} c_{n 2} \cdots c_{n k} \\
w_{i k} & w_{11} w_{12} \cdots w_{1 k} & w_{21} w_{22} \cdots w_{2 k} & \cdots & w_{n 1} w_{n 2} \cdots w_{n k}
\end{array}\right]
$$

Where $c_{i k}$ stands for the k-th secondary influential factor which belongs to the i-th main influential factor in hierarchy model of automobile demand, we use $w_{i k}(i=1,2 \cdots, n ; k=1,2 \cdots p)$ to express their corresponding right.

\subsubsection{Correlation Degree Eigenmatrix}

By correlation transformation of the corresponding degree of membership of automobile demand forecasting system, we get the respective correlation coefficient to construct the eigenmatrix of the correlation coefficient, written as $R_{\xi}$, namely

$$
R_{\xi}=\left[\begin{array}{ccccc} 
& M_{1} & M_{2} & \cdots & M_{m} \\
c_{1} & \xi_{11} & \xi_{21} & \cdots & \xi_{m 1} \\
c_{2} & \xi_{12} & \xi_{22} & \cdots & \xi_{m 2} \\
\vdots & \vdots & \vdots & \cdots & \vdots \\
c_{n} & \xi_{1 n} & \xi_{2 n} & \cdots & \xi_{m n}
\end{array}\right]
$$




\section{Predictive Learning Algorithm Based on Extension Correlation Evaluation and Dynamic Continuous Time Model}

\subsection{Correlation Transformation Algorithm}

It is evident (11) that the degrees of membership about each influential feature are quite dispersive. That is adverse for overall factors comparison. So it is necessary for us to focus on a value. Generally we use weighted centralized processing (Cai, Yang, Chen, \& Li, 2008). Here, we have a new method to determine the weight, i.e. summate characteristic values of factors in different moments, and then implement normalization process to the characteristic values of each factor. Then we can obtain the correlation coefficient of every factor, center upon the automobile sales, figure up the distance between factors in each moment and car sales, and finally do the mean treatment to distance in each moment of the same factor, namely the correlation degrees between the demand for automobiles and various factors. The steps are as follows:

Step 1 Obtain the arithmetic mean value of each factor, namely:

$$
\begin{array}{r}
M_{1} \frac{1}{m}\left(\xi_{11}+\xi_{12}+\cdots+\xi_{1 m}\right)=\frac{1}{m} \sum_{j=1}^{m} \xi_{1 j} \\
M_{2} \frac{1}{m}\left(\xi_{21}+\xi_{22}+\cdots \xi_{2 m}\right)=\frac{1}{m} \sum_{j=1}^{m} \xi_{2 j} \\
M_{n} \frac{1}{m}\left(\xi_{n 1}+\xi_{n 2}+\cdots+\xi_{n m}\right)=\frac{1}{m} \sum_{j=1}^{m} \xi_{n j}
\end{array}
$$

Step2 Implement normalization treatment to each factor's characteristic degree

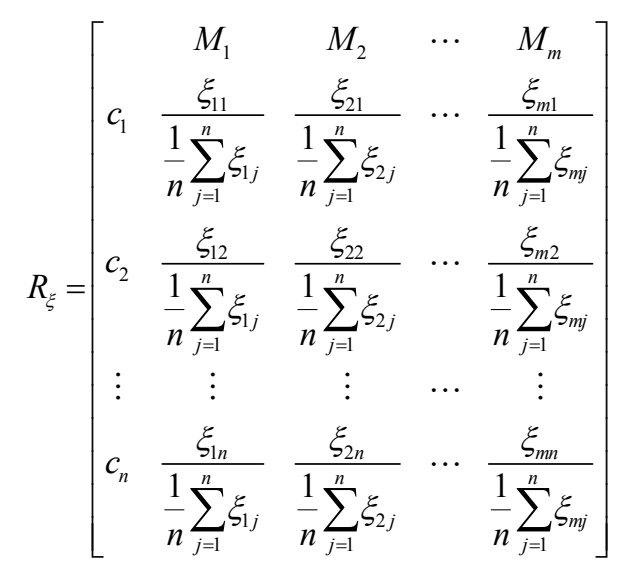

Step3 Center on $M_{k}, 1 \leq k \leq m$, calculate distance between $M_{i}, i=1,2, \cdots, k-1, k+1, \cdots, m$ and $M_{k}, 1 \leq k \leq m \quad d_{i k}\left(M_{i}, M_{k}\right), i=1,2, \cdots, k-1, k+1, \cdots m ; 1 \leq k \leq m$. 


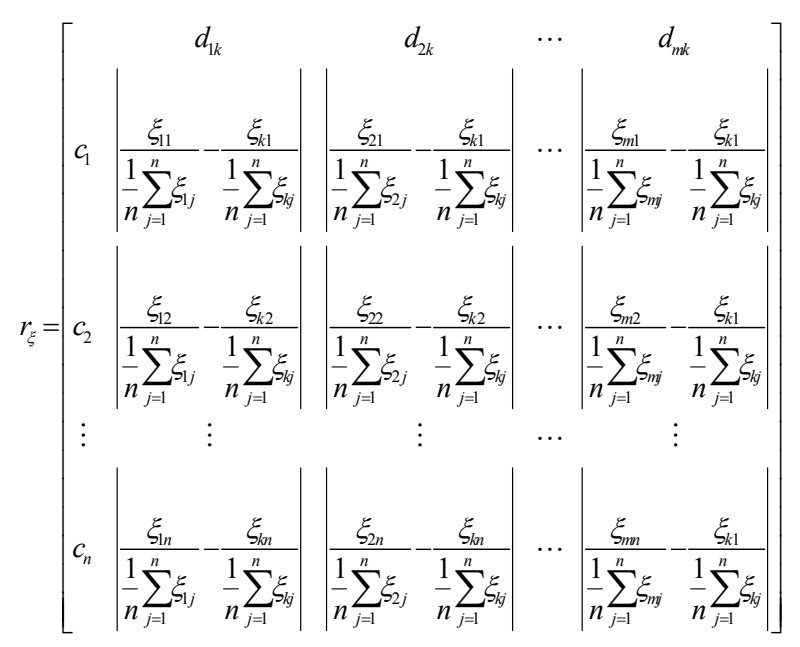

Step4 Comprise correlation degree $r_{k}$ between automobile demand influential factors $M_{i}, i=1,2, \cdots, k-1, k+1, \cdots, m$ and factor $M_{k}, 1 \leq k \leq m$

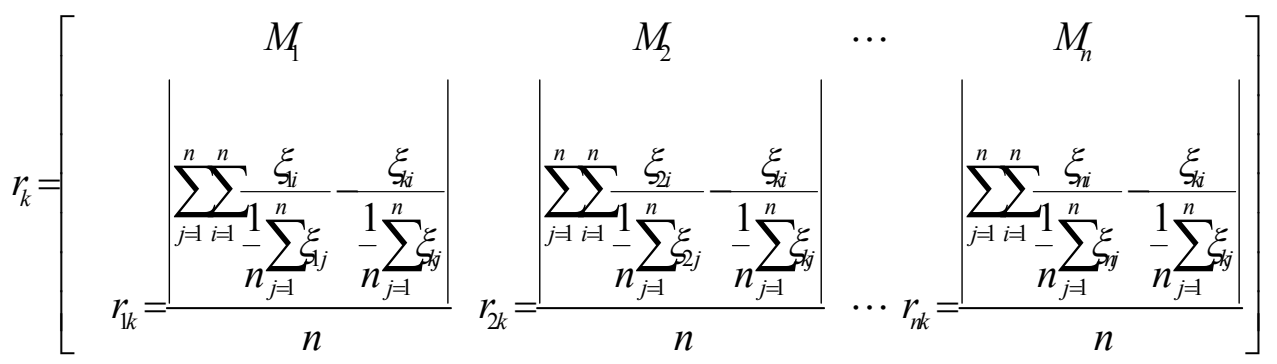

Where $r_{i k}, i=1,2, \cdots, k-1, k+1, \cdots, m$ represent correlation degrees between i-th influential factors of automobile demand forecast system and the k-th item.

\subsection{Demand Status Analyses Based on Extension Theory and Hierarchy Evaluation Model}

Step1 Analysis of the relationship between factors in the automobile demand system, establish the hierarchical structure of the system;

Step2 Process time series of the various influential factors with algorithm 3.1, then get correlation degrees between factors and auto demand;

Step3 Calculate correlation degrees between the various factors and demand target, then sort;

Step4 Study the impact of various factors on the demand for cars with correlation degree as the evaluation of indicators.

\subsection{Solution of Predictive Model (Choose Model 2 as a Sample)}

Step 1 Process series $\left\{x_{1}{ }^{(0)}(t)\right\}$ by means of accumulated operation

$$
x_{1}{ }^{(1)}(k)=\sum_{t=1}^{k} x_{1}{ }^{(0)}(t)
$$

Step2 Implement mean value treatment to series $\left\{x_{1}{ }^{(1)}(t)\right\}$, the model turns into

$$
x_{1}^{(0)}(k)+\frac{\mathrm{a}}{2}\left(x_{1}^{(1)}(k)+x_{1}^{(1)}(k-1)\right)=\mu
$$

Step3 Solve (16) by least square method 


$$
\begin{gathered}
\hat{\mathrm{a}}=\left(B^{T} B\right)^{-1} B^{T} Y \\
\text { Where } B=\left[\begin{array}{cr}
-1 / 2\left(x_{1}^{(1)}(2)+x_{1}{ }^{(1)}(1)\right) & 1 \\
-1 / 2\left(x_{1}{ }^{(1)}(3)+x_{1}{ }^{(1)}(2)\right) & 1 \\
\ldots & \cdots \\
\cdots & \ldots \\
-1 / 2\left(x_{1}{ }^{(1)}(n)+x_{1}{ }^{(1)}(n-1)\right) & 1
\end{array}\right] \\
\hat{\mathrm{a}}=(\mathrm{a}, \mu)^{\mathrm{T}} \\
Y=\left(x_{1}{ }^{(0)}(2), x_{1}{ }^{(0)}(3), \ldots \ldots, x_{1}{ }^{(0)}(n)\right)^{T}
\end{gathered}
$$

Step4 After we get a, $\mu$, the answer to model (2) is

$$
x_{1}^{(1)}(t)=\left(x_{1}^{(1)}(0)-\frac{\mu}{\mathrm{a}}\right) e^{-\mathrm{a} t}+\frac{\mu}{\mathrm{a}}
$$

Step5 Implement inverse accumulated operation

$$
x_{1}^{(0)}(t)=x_{1}^{(1)}(t+1)-x_{1}^{(1)}(t)
$$

Then we can get the predicted value

\subsection{System Prediction Value of K Moments in the Future}

Step 1 Process measured random sequence with accumulated operation and mean value treatment

Step2 If $\left\{x^{(0)}(t)\right\}$ is linear dynamic system with single factor sequence, establish forecast model according to formula (2),or if it is linear dynamic system with two-factor sequence, establish forecast model according to formula (3), or if it is linear dynamic system with three-factor sequence, establish forecast model according to formula (4);

Step3 Solve the model with 3.3;

Step 4 Get the solutions by inverse accumulated operation, and then the results are predicted values of nonstationary sequence.

\subsection{Analysis and Forecast of Automobile Short-Term Demand}

Step 1 Qualitatively analyze influential factor with algorithm 3.1, and then obtain correlation coefficients of each demand influential factor;

Step 2 Process primitive correlation coefficient by utilizing algorithm 3.2, get the weight of each demand influential factor;

Step 3 Analyze and forecast automobile short-term demand with algorithm 3.1, 3.3, 3.4 .

\section{Example Analyses}

Analyze the model in the paper by taking car demand forecast analysis which comes from a certain area as an example. Set automotive history sales during 2000-2012 as the primitive data, implement correlation analyses to the auto demand influential factors, the results can help the government to make macro-control to automobile industry and automobile manufacturing enterprises to make production plan. The region's car sales and influential factors of automobile demand statistics are in Table 1. 
Table 1. Numerical table of automobile demand influential factors

\begin{tabular}{|c|c|c|c|c|c|c|c|c|c|}
\hline year & $\begin{array}{l}\text { total car sales } \\
\text { (10 thousand) }\end{array}$ & $\begin{array}{l}\text { gross domestic } \\
\text { product (GDP) } \\
\text { (one } \\
\text { hundred million } \\
\text { Yuan) }\end{array}$ & $\begin{array}{l}\text { per capita } \\
\text { GDP } \\
\text { (Yuan) }\end{array}$ & $\begin{array}{l}\text { proportion of the } \\
\text { third industry } \\
\text { GDP (\%) }\end{array}$ & $\begin{array}{l}\text { social fixed } \\
\text { assets } \\
\text { investment } \\
\text { (one hundred } \\
\text { million Yuan) }\end{array}$ & $\begin{array}{l}\text { retail sales of } \\
\text { social } \\
\text { consumption } \\
\text { (one hundred } \\
\text { million Yuan) }\end{array}$ & $\begin{array}{l}\text { premium (one } \\
\text { hundred } \\
\text { million Yuan) }\end{array}$ & $\begin{array}{l}\text { per capita } \\
\text { annual income } \\
\text { (Yuan) }\end{array}$ & $\begin{array}{l}\text { urban and rural } \\
\text { residents' } \\
\text { year-end savings } \\
\text { balance } \\
\text { (one hundred } \\
\text { million Yuan) }\end{array}$ \\
\hline 2000 & 205.42 & 99214.6 & 7858 & 39.02 & 32917.73 & 39105.7 & 1596 & 3711.83 & 54332 \\
\hline 2001 & 249.96 & 109655.2 & 8622 & 40.46 & 37213.49 & 43055.4 & 2109.0 & 4058.54 & 73762.0 \\
\hline 2002 & 289.67 & 120332.7 & 9398 & 41.47 & 43499.91 & 48135.9 & 3054.0 & 4518.90 & 86911.0 \\
\hline 2003 & 358.36 & 135822.8 & 10542 & 41.23 & 55566.60 & 52516.3 & 3880.0 & 4993.22 & 103617.0 \\
\hline 2004 & 423.65 & 159878.3 & 12336 & 40.38 & 70477.40 & 59501.0 & 4714.0 & 5644.62 & 119555.4 \\
\hline 2005 & 572.90 & 184937.4 & 14185 & 40.51 & 88773.60 & 67176.6 & 4932.0 & 6366.56 & 141051.0 \\
\hline 2006 & 721.60 & 216314.4 & 16500 & 40.94 & 109998.16 & 76410.0 & 5640.0 & 7174.73 & 161587.3 \\
\hline 2007 & 879.15 & 265810.3 & 20169 & 41.89 & 137323.94 & 89210.0 & 7036.0 & 8475.04 & 172534.2 \\
\hline 2008 & 938.05 & 314045.4 & 23708 & 41.82 & 172828.40 & 114830.1 & 9784.0 & 9794.87 & 217885.4 \\
\hline 2009 & 1364.50 & 340506.9 & 25575 & 43.36 & 224598.77 & 132678.4 & 11137.0 & 10753.98 & 260771.7 \\
\hline 2010 & 1806.19 & 401512.8 & 30015 & 43.24 & 251683.77 & 156998.4 & 14528.0 & 14811.72 & 303302.5 \\
\hline 2011 & 1850.51 & 473104.1 & 35198 & 43.37 & 311485.13 & 183918.6 & 14339.3 & 15694.32 & 343635.9 \\
\hline 2012 & 1930.64 & 519470.1 & 38459 & 44.65 & 374694.74 & 210307.0 & 15487.9 & 17145.76 & 399551.8 \\
\hline
\end{tabular}

Data source: "China statistical yearbook", 2000-2013.

In practice, there are many car demand influential factors, including gross domestic product (GDP), per capita GDP, proportion of the third industry GDP, social fixed assets investment, retail sales of social consumption, per capita annual income, urban and rural residents' year-end savings balance, premium and other factors, this paper chooses the above factors to analysis their influence to car demand. Process data in Table 1 according to algorithm 4.1 and 4.2, respectively we get Table 2 and Table 3 and Table 4. Table 2 presents the correlation coefficients of demand factors system in different moments, Table 3 shows the distance from factors to car sales. Correlation coefficients for all impact factors with regard to car sales are shown in Table 4. Table 5 represents correlation degrees among factors relative to car sales.

Table 2. Factors' correlation transformation processing

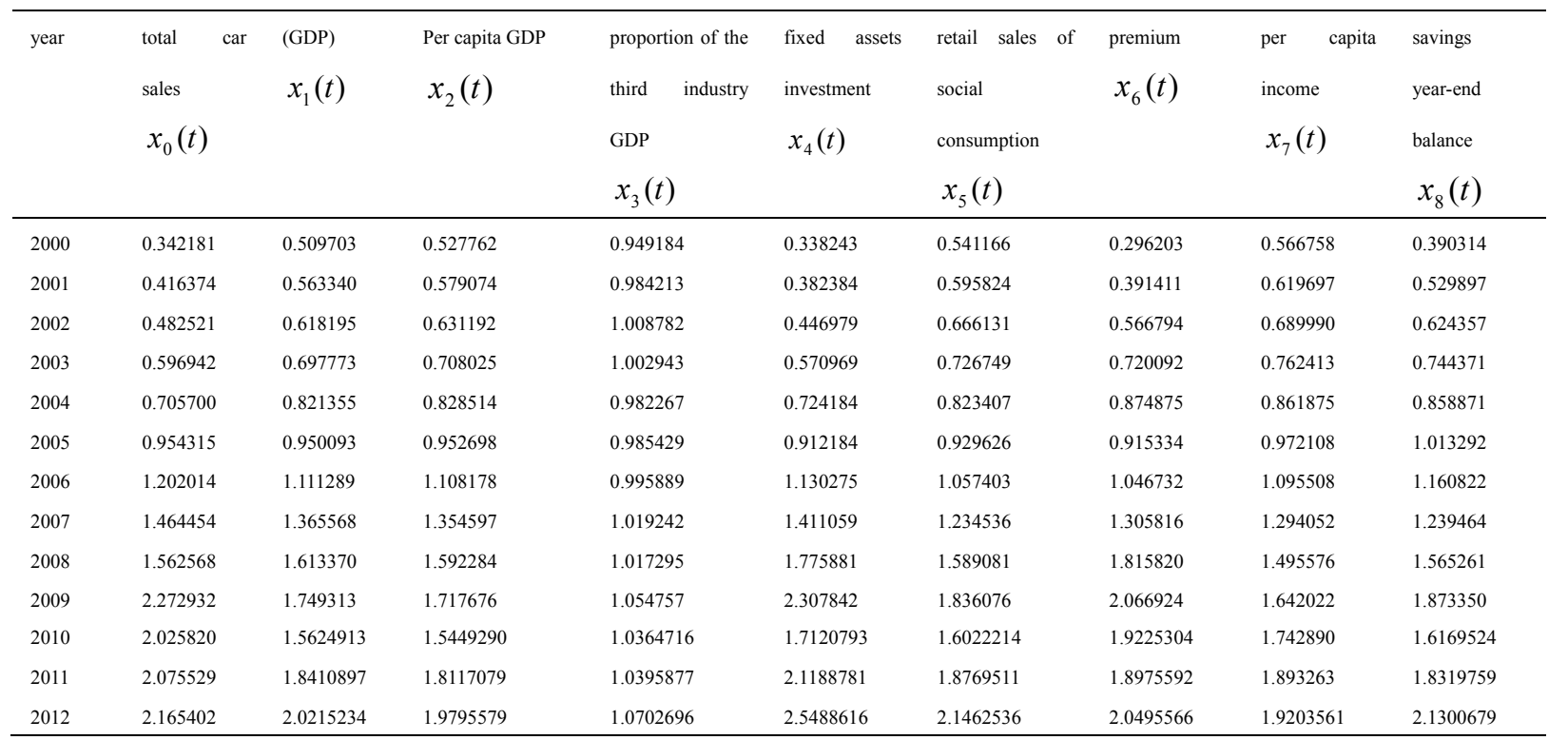


Table 3. Distance between factors and car sales

\begin{tabular}{lllllllll}
\hline year & $\Delta_{01}(t)$ & $\Delta_{02}(t)$ & $\Delta_{03}(t)$ & $\Delta_{04}(t)$ & $\Delta_{05}(t)$ & $\Delta_{06}(t)$ & $\Delta_{07}(t)$ & $\Delta_{08}(t)$ \\
\hline 2000 & 0.167522 & 0.185581 & 0.607003 & 0.003938 & 0.198985 & 0.045978 & 0.224578 & 0.048133 \\
2001 & 0.146967 & 0.162700 & 0.567839 & 0.033990 & 0.179450 & 0.024963 & 0.203324 & 0.113523 \\
2002 & 0.135673 & 0.148670 & 0.526260 & 0.035542 & 0.183610 & 0.084273 & 0.207468 & 0.141836 \\
2003 & 0.100831 & 0.111083 & 0.406001 & 0.025973 & 0.129807 & 0.123150 & 0.165471 & 0.147429 \\
2004 & 0.115655 & 0.122815 & 0.276567 & 0.018484 & 0.117707 & 0.169175 & 0.156176 & 0.153171 \\
2005 & 0.004221 & 0.001617 & 0.031114 & 0.042130 & 0.024689 & 0.038981 & 0.017793 & 0.058977 \\
2006 & 0.090725 & 0.093835 & 0.206125 & 0.071738 & 0.144610 & 0.155282 & 0.106506 & 0.041191 \\
2007 & 0.098886 & 0.109857 & 0.445213 & 0.053396 & 0.229918 & 0.158638 & 0.170403 & 0.224991 \\
2008 & 0.050802 & 0.029717 & 0.545272 & 0.213313 & 0.026514 & 0.253252 & 0.066992 & 0.002693 \\
2009 & 0.523619 & 0.555255 & 1.218175 & 0.034911 & 0.436856 & 0.206008 & 0.630909 & 0.399581 \\
2010 & 0.4633287 & 0.4808910 & 0.9893485 & 0.3137408 & 0.4235986 & 0.1032897 & 0.4088677 \\
2011 & 0.2344397 & 0.2638214 & 1.0359416 & 0.0433488 & 0.1985782 & 0.1779701 & 0.2435534 \\
2012 & 0.1438796 & 0.1858453 & 1.0951334 & 0.3834586 & 0.0191494 & 0.1158464 & & 0.035335 \\
\hline
\end{tabular}

Table 4. Correlation coefficients among factors

\begin{tabular}{|c|c|c|c|c|c|c|c|c|}
\hline year & $\xi_{01}(t)$ & $\xi_{02}(t)$ & $\xi_{03}(t)$ & $\xi_{04}(t)$ & $\xi_{05}(t)$ & $\xi_{06}(t)$ & $\xi_{07}(t)$ & $\xi_{08}(t)$ \\
\hline 2000 & 0.786373 & 0.768503 & 0.502187 & 0.996214 & 0.755755 & 0.932281 & 0.732554 & 0.929223 \\
\hline 2001 & 0.807753 & 0.791286 & 0.518898 & 0.949659 & 0.774477 & 0.963180 & 0.751719 & 0.845137 \\
\hline 2002 & 0.820001 & 0.805937 & 0.537901 & 0.947373 & 0.770414 & 0.880790 & 0.747903 & 0.813271 \\
\hline 2003 & 0.860246 & 0.848000 & 0.601627 & 0.961648 & 0.826512 & 0.834026 & 0.788455 & 0.807259 \\
\hline 2004 & 0.842650 & 0.834408 & 0.689552 & 0.973124 & 0.840271 & 0.784703 & 0.798032 & 0.801178 \\
\hline 2005 & 0.995754 & 1.000000 & 0.953926 & 0.937789 & 0.963597 & 0.942346 & 0.974196 & 0.914140 \\
\hline 2006 & 0.872670 & 0.868808 & 0.749136 & 0.897006 & 0.810278 & 0.798966 & 0.853424 & 0.939143 \\
\hline 2007 & 0.786373 & 0.768503 & 0.502187 & 0.996214 & 0.755755 & 0.932281 & 0.732554 & 0.929223 \\
\hline 2008 & 0.807753 & 0.791286 & 0.518898 & 0.949659 & 0.774477 & 0.963180 & 0.751719 & 0.845137 \\
\hline 2009 & 0.820001 & 0.805937 & 0.537901 & 0.947373 & 0.770414 & 0.880790 & 0.747903 & 0.813271 \\
\hline 2010 & 0.38001623 & 0.388703907 & 0.368026275 & 0.384428269 & 0.36347096 & 0.545756953 & 0.723199 & 0.39094783 \\
\hline 2011 & 0.56663028 & 0.556027588 & 0.357197476 & 0.826606082 & 0.56277135 & 0.409574148 & 0.786101 & 0.53521372 \\
\hline 2012 & 0.70326967 & 0.657734929 & 0.344326489 & 0.337832202 & 1 & 0.516861264 & 0.758231 & 1 \\
\hline
\end{tabular}

Table 5. Correlation degrees of impact factors

\begin{tabular}{lllllll}
\hline correlation degree & $\gamma_{01}$ & $\gamma_{02}$ & $\gamma_{03}$ & $\gamma_{04}$ & $\gamma_{05}$ & $\gamma_{06}$ \\
\hline & 0.7099 & 0.7098 & 0.5699 & 0.7858 & 0.6973 & 0.6351 \\
\hline
\end{tabular}

Illustrate Table 5 with Figure 2., which represents correlation degrees among factors with regard to automobile sales, where we can find that $\gamma_{04}>\gamma_{08}>\gamma_{01}>\gamma_{02}>\gamma_{05}>\gamma_{07}>\gamma_{06}>\gamma_{03}$, hence fixed assets investment has the closest correlation relationship with automobile sales, the second closet is premium. Third industry GDP has the minimum influence. 


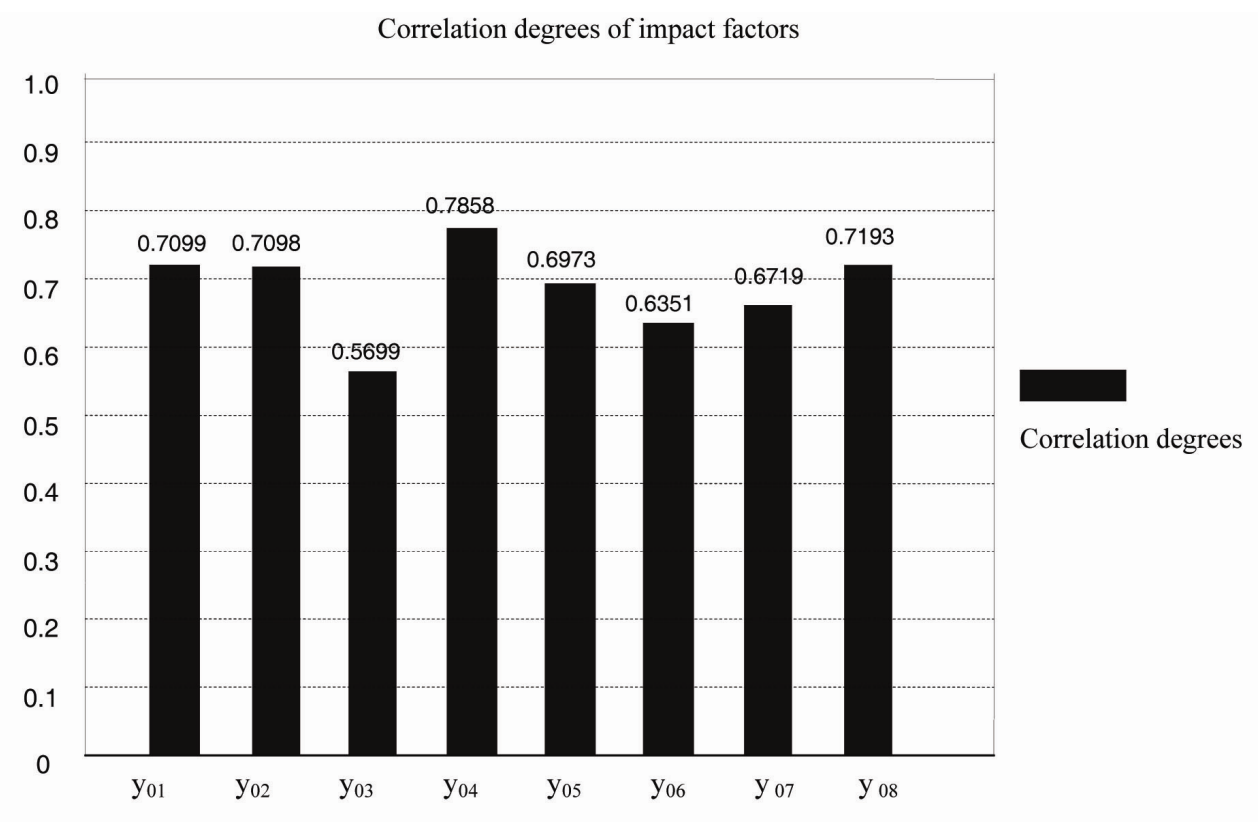

Figure 2. Correlation degrees of impact factors

According to the forecast model and method proposed by this paper, forecast continuous data of car demand during 2000-2012, and compare them with the existing data, the result is shown in Table 6.

Table 6. Comparison between car demand forecast values and actual values during 2000-2012

\begin{tabular}{lllll}
\hline time & actual value & predicted value & absolute error & relative error \\
\hline 2000 & 205.42 & 205.42 & 0.00 & 0.0000 \\
2001 & 249.96 & 234.90 & 15.06 & 0.0602 \\
2002 & 289.67 & 289.44 & 0.23 & 0.0008 \\
2003 & 358.36 & 358.17 & 0.19 & 0.0005 \\
2004 & 423.65 & 443.22 & 19.57 & 0.0462 \\
2005 & 572.90 & 548.46 & 24.44 & 0.0427 \\
2006 & 721.60 & 678.70 & 42.90 & 0.0595 \\
2007 & 879.15 & 839.86 & 39.29 & 0.0447 \\
2008 & 938.05 & 1039.28 & 101.23 & 0.1079 \\
2009 & 1364.50 & 1286.07 & 78.43 & 0.0575 \\
2010 & 1806.19 & 176124 & 44.95 & 0.0249 \\
2011 & 1850.51 & 1792.68 & 57.83 & 0.0313 \\
2012 & 1930.64 & 1889.12 & 41.52 & 0.0215 \\
\hline
\end{tabular}

Predicted results based on the above model are given in Table 6 reflects that with the method in this paper we have a fine fitting degree between predicted series and primitive series during 2000-2012. Except for relative errors in statistics of year 2008, the others are controlled within $6 \%$, that means this model can forecast short-term time series accurately. A big reason of 2008 prediction error is the financial crisis which makes great volatility to automobile demand. In 2009 , it returns to normal predictive error.

The above analysis result shows that we can make an effective assessment on demand system, and simultaneously achieve the purpose of car demand control through the related economic policies.

\section{Conclusion}

Automotive industry has become an important part of China's industry, prediction model proposed by this paper has solved difficult problems that short-term forecast in traditional prediction method cannot handle with; it is also able to fit uncertain car demand changes with higher precision. By using the prediction model to forecast car demand in the coming three years, we can see that car demand will grow continuously, and the growth rate will also increase year by year. 
At the same time, the extensive evaluation model set up in this paper provides automobile enterprises and government agencies with a feasible and effective method for car demand forecast analysis. The model analyses main factors of car demand influential, and sort them according to the extent of the effects. We found that among various influential factors, fixed assets investment and the premium affect a lot. However, the third industry GDP has little effect. These analyses will help the government to make macro-control to automobile demand and the same to the automobile manufacturers to make production plan.

\section{References}

Bao, Y. J. (2011). Integrated Forecast on Car Ownership Rate of Urban Dwellers in China: Based on Different Income Levels. Technology Economics, 30, 87-90.

Cai, W. (1999). Extension theory and its application. Chinese Science Bulletin, 44, 1538-1548. http://dx.doi.org/10.1007/BF02886090

Cai, W., Yang, C. Y., Chen, W. W., \& Li, X. S. (2008). Extension set and extension data mining. Science Press.

Feng, B. (2004). Key Factors Analysis of Chinese Automobile Brand Development. Tianjin Auto, 4, 6-9.

Liu, Z. (2009). Application of Grey System Theory for Port Container Throughput Forecasting. Hefei University of Technology, 2009.

Su, M. (2010). Influential Factors, Trend and Distributional Change of Cars'Demand-Empirical Analysis Based on the Household Private Cars Ownership. Journal of Shanxi Finance and Economics University, 32, 43-49.

Wang, L. L., \& Ma, X. (2006). Analysis on Econometric Model of Demand of Chinese Civil Automobile. Journal of Shanxi Economic Management Institute, 14, 16-18.

Zhao, S. P. (1991). A New Method of Establishing Time Continuous Model of Dynamic System. Systems Engineering-theory \& Practice, 5, 4-9.

Zuo, X. Y., \& Gao, S. (2011). Forecasting system analysis for automotive market quantity demand based on petri net. Journal of Shandong University of Technology(Natural Science Edition), 25, 53-57.

\section{Copyrights}

Copyright for this article is retained by the author(s), with first publication rights granted to the journal.

This is an open-access article distributed under the terms and conditions of the Creative Commons Attribution license (http://creativecommons.org/licenses/by/3.0/). 